\title{
Chiral metamaterials with negative refractive index based on four "U" split ring resonators
}

Zhaofeng Li, Rongkuo Zhao, Thomas Koschny, Maria Kafesaki, Kamil Boratay Alici, Evrim Colak, Humeyra Caglayan, Ekmel Ozbay, and C. M. Soukoulis

Citation: Appl. Phys. Lett. 97, 081901 (2010);

View online: https://doi.org/10.1063/1.3457448

View Table of Contents: http://aip.scitation.org/toc/apl/97/8

Published by the American Institute of Physics

\section{Articles you may be interested in}

Broadband polarization transformation via enhanced asymmetric transmission through arrays of twisted complementary split-ring resonators

Applied Physics Letters 99, 221907 (2011); 10.1063/1.3664774

$90^{\circ}$ polarization rotator using a bilayered chiral metamaterial with giant optical activity

Applied Physics Letters 96, 203501 (2010); 10.1063/1.3429683

Dual-band asymmetric transmission of linear polarization in bilayered chiral metamaterial Applied Physics Letters 102, 191905 (2013); 10.1063/1.4805075

Complementary chiral metamaterials with giant optical activity and negative refractive index Applied Physics Letters 98, 161907 (2011); 10.1063/1.3574909

Optical activity in extrinsically chiral metamaterial

Applied Physics Letters 93, 191911 (2008); 10.1063/1.3021082

Compact circular polarizer based on chiral twisted double split-ring resonator

Applied Physics Letters 102, 103503 (2013); 10.1063/1.4794940

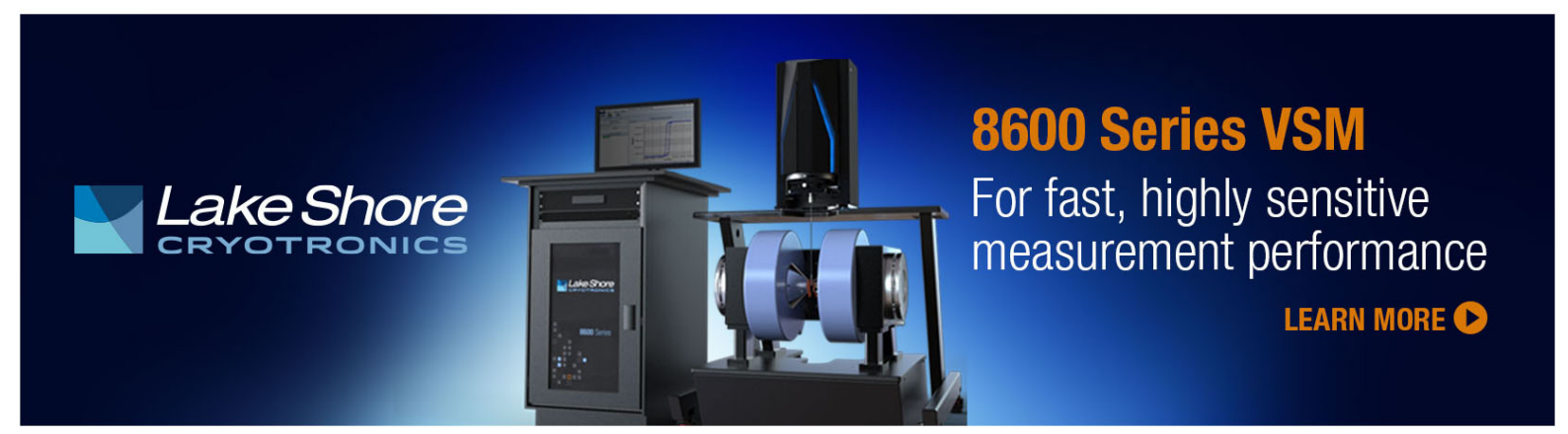




\title{
Chiral metamaterials with negative refractive index based on four " $U$ " split ring resonators
}

\author{
Zhaofeng Li, ${ }_{1}$ Rongkuo Zhao, ${ }^{2,3}$ Thomas Koschny, ${ }_{1,5}^{2,4}$ Maria Kafesaki, ${ }^{4}$ Kamil Boratay Alici, ${ }^{1}$ \\ Evrim Colak, ${ }^{1}$ Humeyra Caglayan, ${ }^{1}$ Ekmel Ozbay, ${ }^{1,5}$ and C. M. Soukoulis ${ }^{2,4, a)}$ \\ ${ }^{1}$ Nanotechnology Research Center and Department of Physics, Bilkent University, Bilkent, \\ 06800 Ankara, Turkey \\ ${ }^{2}$ Ames Laboratory and Department of Physics and Astronomy, Iowa State University, Ames, \\ Iowa 50011, USA \\ ${ }^{3}$ Applied Optics Beijing Area Major Laboratory, Department of Physics, Beijing Normal University, \\ Beijing 100875, China \\ ${ }^{4}$ Institute of Electronic Structure and Laser, FORTH, and Department of Materials Science and Technology, \\ University of Crete, 71110 Heraklion, Crete, Greece \\ ${ }^{5}$ Department of Electrical and Electronics Engineering, Bilkent University, Bilkent, 06800 Ankara, Turkey
}

(Received 5 February 2010; accepted 4 June 2010; published online 23 August 2010)

\begin{abstract}
A uniaxial chiral metamaterial is constructed by double-layered four " $U$ " split ring resonators mutually twisted by $90^{\circ}$. It shows a giant optical activity and circular dichroism. The retrieval results reveal that a negative refractive index is realized for circularly polarized waves due to the large chirality. The experimental results are in good agreement with the numerical results. (C) 2010 American Institute of Physics. [doi:10.1063/1.3457448]
\end{abstract}

Chiral metamaterials (CMMs) have attracted much attention ever since it was predicted that negative refraction can be achieved by a chiral route. ${ }^{1,2}$ In fact, CMMs also have other interesting properties, such as the giant optical activity and circular dichroism, which may find their way into optical applications. $^{3-8}$ As an artificial metamaterial, CMM lacks any mirror symmetry so that the cross-coupling between the electric and magnetic fields exists at the resonance. The degeneracy of the two circularly polarized waves is thereby broken, i.e., right circularly polarized $(\mathrm{RCP},+)$ waves and left circularly polarized $(\mathrm{LCP},-)$ waves have different refractive indices. The chirality parameter $\kappa$ describes the strength of the cross-coupling effect, so that the constitutive relations of a chiral medium is given by

$$
\left(\begin{array}{l}
D \\
B
\end{array}\right)=\left(\begin{array}{cc}
\varepsilon_{0} \varepsilon & -i \kappa / c \\
i \kappa / c & \mu_{0} \mu
\end{array}\right)\left(\begin{array}{l}
E \\
H
\end{array}\right)
$$

where $\varepsilon_{0}$ and $\mu_{0}$ are the permittivity and permeability of vacuum. $\varepsilon$ and $\mu$ are the relative permittivity and permeability of the chiral medium. $c$ is the speed of light in vacuum. Assuming a time dependence of $e^{-i \omega t}$, the $\mathrm{RCP}(+)$ wave and $\mathrm{LCP}(-)$ wave are defined as $E^{ \pm}=(1 / 2) E_{0}(\hat{x} \mp i \hat{y}) .{ }^{9}$ The refractive indices for RCP and LCP waves can be expressed as $n_{ \pm}=n \pm \kappa,{ }^{10}$ where $n=\sqrt{\varepsilon \mu}$. When $\kappa$ is large enough, either $n_{+}$or $n_{-}$becomes negative. At the same time, both RCP and LCP waves have the same impedance of $z / z_{0}=\sqrt{\mu / \varepsilon}$, where $z_{0}$ is the impedance of the vacuum.

Since the beginning of the proposal of metamaterials, split ring resonators (SRRs) have played an important role in achieving negative permeability. Experiments showed that SRRs can be used as magnetic metamaterials even at infrared and visible frequencies. ${ }^{1-13}$ Furthermore, a magnetic dimer can be formed by stacking two mutually twisted SRRs, and an array of these magnetic dimers can possess optical activity. ${ }^{14,15}$ However, due to the lack of the rotational sym-

${ }^{a)}$ Electronic mail: soukoulis@ameslab.gov. metry, the optical activity is sensitive to the linear polarization of the incident wave. To eliminate this shortage, we propose here a design in which the U-shaped SRR pairs are arranged in C4 symmetry (see Fig. 1). Thus, the constructed CMM is effectively uniaxial for the normal incidence wave. A related retrieval procedure ${ }^{6,16,17}$ can then be applied to calculate the effective parameters for our CMMs.

Figure 1 shows the schematics of the CMMs we study. The unit cell of the CMM structure consists of doublelayered four "U" SRRs (Four-U-SRRs) patterned on opposite sides of an FR-4 board. This structure has been mentioned theoretically ${ }^{18}$ and is discussed in another papers. ${ }^{19,20}$ The relative dielectric constant of the FR-4 board is 4.0 with a dielectric loss tangent of 0.025 . The two layers of Four-USRRs are mutually twisted by $90^{\circ}$. The Four-U-SRRs are arranged so that the CMM structure possesses $\mathrm{C} 4$ symmetry. The dimensions of the unit cell and the photo of our experimental sample are shown in Fig. 1 and the caption.

In order to study the behavior of the chiral structure, we conducted numerical simulations and experiments. The simulation works were carried out by using CST MICROWAVE STUDIO (Computer Simulation Technology GmbH, Darmstadt, Germany), wherein the finite integration technique was (a)

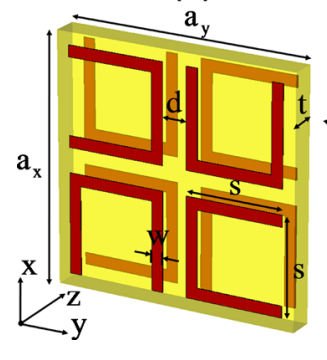

(b)

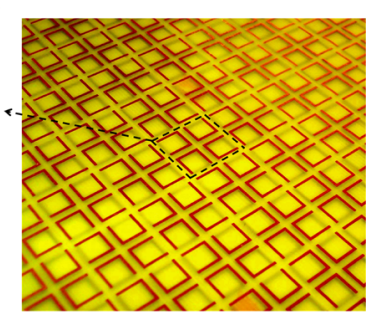

FIG. 1. (Color online) (a) Schematic of a unit cell of the CMMs consisting of four "U" SRRs. (b) A photo of the experimental sample. The geometric parameters are given by $\mathrm{a}_{\mathrm{x}}=\mathrm{a}_{\mathrm{y}}=15 \mathrm{~mm}, \mathrm{t}=1.6 \mathrm{~mm}, \mathrm{~d}=1.5 \mathrm{~mm}, \mathrm{w}$ $=0.7 \mathrm{~mm}$, and $\mathrm{s}=6 \mathrm{~mm}$. The copper has a thickness of $0.03 \mathrm{~mm}$. 


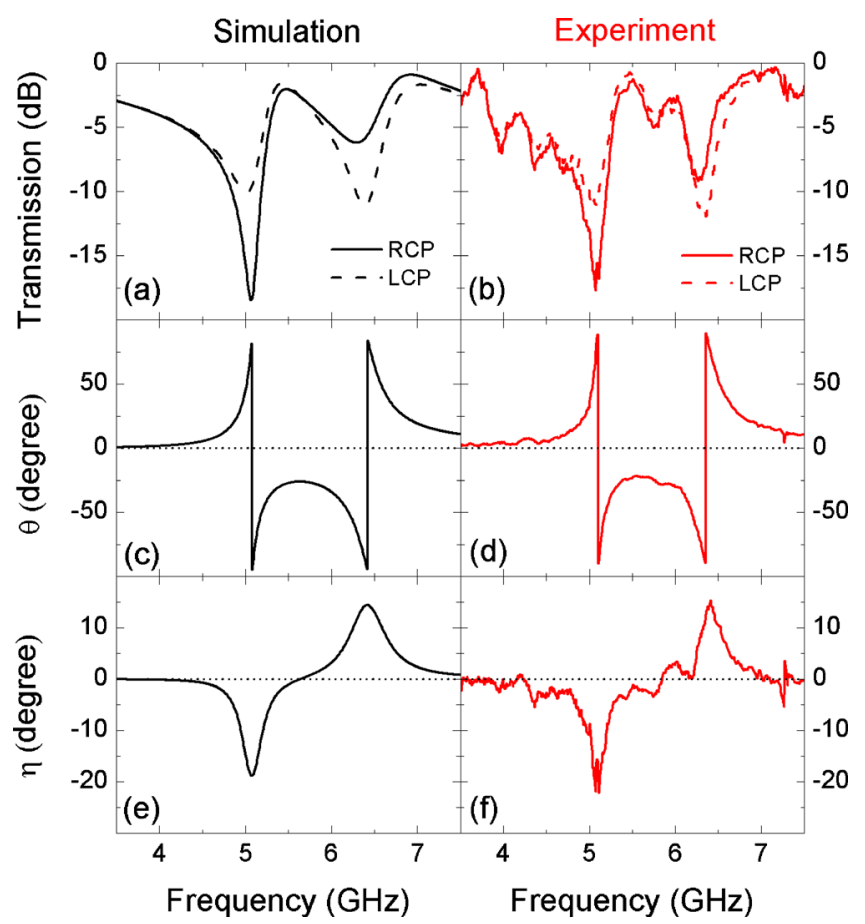

FIG. 2. (Color online) Simulation and experimental results for the CMM. (a) and (b) are the transmission spectra for RCP and LCP waves. (c) and (d) are the polarization azimuth rotation angle $\theta$. (e) and (f) are the ellipticity angle $\eta$ of the transmitted wave.

applied. The periodic boundary conditions were applied to the $x$ and $y$ directions and the absorbing boundary conditions were applied to the $z$ direction. In the experiment, we fabricated the chiral structures with a dimension of 20 by 20 unit cells. The transmission coefficient was measured by an HP8510C network analyzer with two standard horn antennas.

In order to study the transmission behaviors of the chiral structure, in our simulation and experimental works, a linearly polarized electromagnetic wave (E field in the $x$ direction) is incident on the chiral structure. On the other side of the structure, we measured the transmitted field in the $x$ and $y$ polarizations $\left(T_{x x}\right.$ and $\left.T_{y x}\right)$. Due to the fourfold rotational symmetry, circular polarization conversion is absent. The transmission of circularly polarized waves can be converted from the linear transmission coefficients $T_{x x}$ and $T_{y x},{ }^{6,7} T^{ \pm}$ $=T_{x x} \pm i T_{y x}$. For the transmitted EM wave, the polarization azimuth rotation angle $\theta$ can be calculated as $\theta=\left[\arg \left(T^{+}\right)\right.$ $\left.-\arg \left(T^{-}\right)\right] / 2$. The ellipticity of the transmitted wave is defined as $\eta=\arctan \left[\left(\left|T^{+}\right|-\left|T^{-}\right|\right) /\left(\left|T^{+}\right|+\left|T^{-}\right|\right)\right],{ }^{9}$ which also measures circular dichroism.

Figures 2(a) and 2(b) show the simulated and measured transmission spectra. There are obvious differences between the transmissions of RCP and LCP waves around the resonances. Around the frequency of $5.1 \mathrm{GHz}$, the transmission of LCP wave is 7-8 dB higher than that of the RCP wave. While around the frequency of $6.3 \mathrm{GHz}$, the transmission of the LCP wave is around 3-4 dB lower than that of the RCP wave. The fluctuations on the experiment curve come from the multiple reflections between the CMM structure and the horn antennas.

Figures 2(c)-2(f) show the simulated and experimental results of the polarization azimuth rotation angle $\theta$ and the ellipticity $\eta$ of the transmitted wave. It can be clearly seen that, in the middle of the two resonant frequencies, the ellip-
Simulation

Experiment

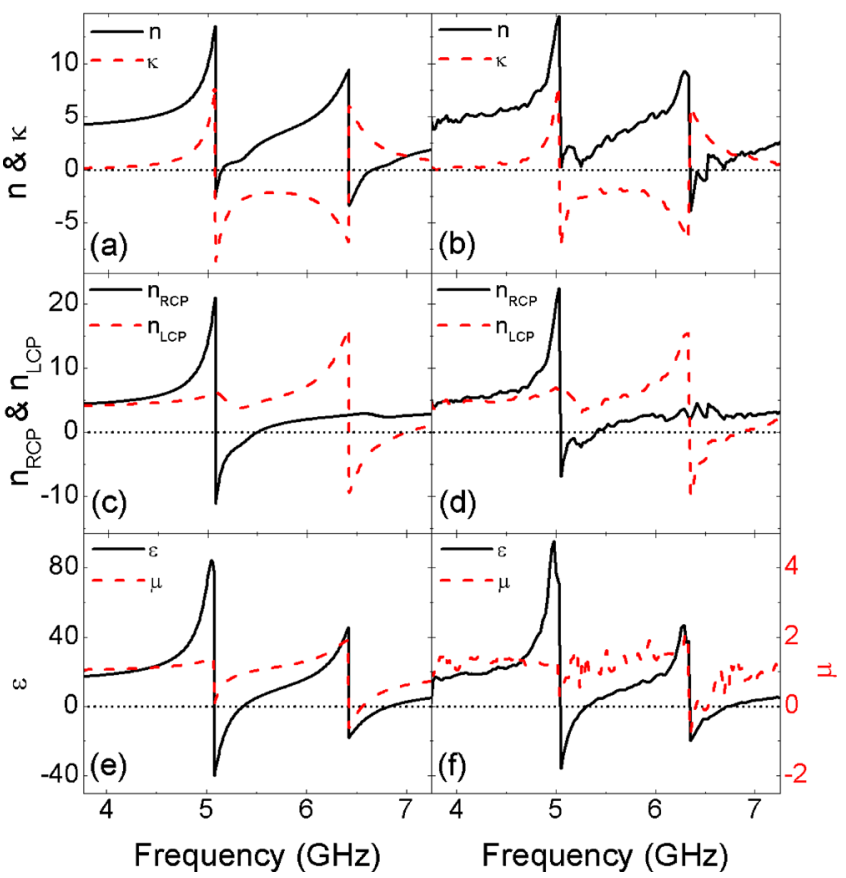

FIG. 3. (Color online) The retrieved effective parameters of the CMMs based on the simulation (left) and experimental (right) data. (a) and (b) show the real parts of the refractive index $n$ and chirality $\kappa$. (c) and (d) show the real parts of the refractive indices for RCP and LCP waves. (e) and (f) show the real parts of the permittivity $\varepsilon$ and permeability $\mu$.

ticity $\eta=0$, where corresponds a pure optical activity effect, i.e., for the linear polarization incident wave, the transmission wave is still the linear polarization but with a rotated angle $\theta$. The azimuth rotation angle $\theta$ is as large as $26^{\circ}$ at 5.5 GHz. The rotation angle $\theta$ per wavelength is $700^{\circ} / \lambda$.

Figure 3 shows the retrieved effective parameters based on the simulation and experimental data of the transmission and reflection for one layer of the CMMs. In the retrieval process, the effective thickness of the CMM is assumed to be $1.8 \mathrm{~mm}$ along the wave propagation direction. The chirality is very large, e.g., it is 2.15 at $\eta=0$. If using the following expression $^{20}$

$$
\kappa=-\frac{\Omega_{\kappa 1} \omega_{\kappa 1} \omega}{\omega^{2}-\omega_{\kappa 1}^{2}+i \Gamma_{\kappa 1} \omega_{\kappa 1} \omega}+\frac{\Omega_{\kappa 2} \omega_{\kappa 2} \omega}{\omega^{2}-\omega_{\kappa 2}^{2}+i \Gamma_{\kappa 2} \omega_{\kappa 2} \omega},
$$

to fit the result of the chirality $\kappa$, it gives us $\Omega_{\kappa 1}=0.22$, $\omega_{\kappa 1}=5.08 \mathrm{GHz}, \Gamma_{\kappa 1}=0.02, \Omega_{\kappa 2}=0.28, \omega_{\kappa 2}=6.42 \mathrm{GHz}$, and $\Gamma_{\kappa 2}=0.03$. Comparing Figs. 3(a) and 3(b) and Figs. 3(c) and $3(\mathrm{~d})$, due to the relation of $n_{ \pm}=n \pm \kappa$, the strong chirality $\kappa$ can push the refractive index of RCP (LCP) wave to be negative around the resonant frequency of 5.1 (6.4) $\mathrm{GHz}$ as shown in Figs. 3(c) and 3(d). However, due to the losses in the dielectric board, the figure of merit $[-\operatorname{Re}(\mathrm{n}) / \operatorname{Im}(\mathrm{n})]$ is only around $1.1(1.3)$ at $5.1(6.4) \mathrm{GHz}$ in the negative index band. By using a lower loss substrate, the imaginary part of the negative index can be reduced. The figure of merit can be larger than 10 (not shown here). In Figs. 3(e) and 3(f), we show the retrieved real parts for the permittivity and permeability of the CMM. Obviously, the $\operatorname{Re}(\mu)$ is always positive throughout the entire frequency range (except the tiny range around $6.4 \mathrm{GHz}$ ), while $\operatorname{Re}(\varepsilon)$ is negative in the frequency ranges above the two resonances. In traditional metamateri- 

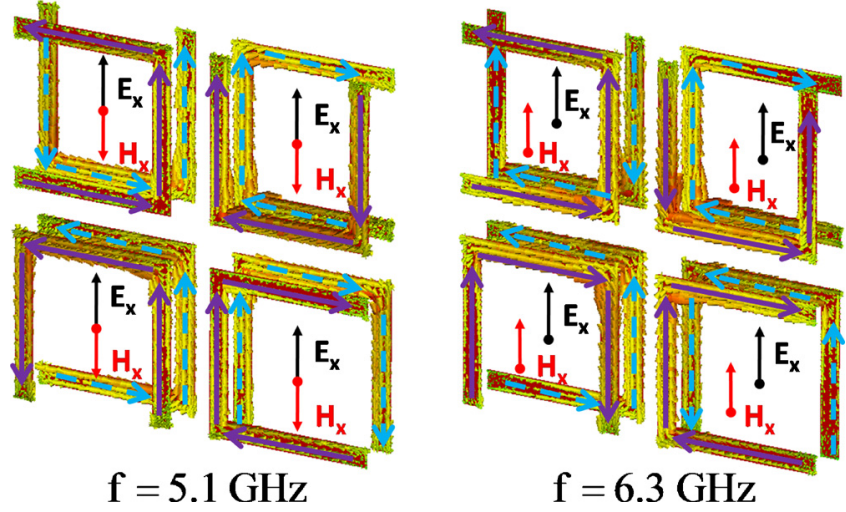

FIG. 4. (Color online) The current distributions driven by the electric field in the $x$ direction at $5.1 \mathrm{GHz}$ and $6.3 \mathrm{GHz}$. The arrows in the middle of the SRRs show the direction of the $x$ component of the induced magnetic field.

als, this will not result in a negative index. Therefore, the negative index of RCP (LCP) wave is actually attributed to the relatively small $\mathrm{n}$ and large chirality $\kappa$.

In order to understand the mechanism of the resonances for our Four-U-SRRs CMM, we study the current modes on the Four-U-SRRs. Figure 4 shows the simulated current modes at 5.1 and $6.3 \mathrm{GHz}$ driven by the electric field in the $x$ direction. At $5.1 \mathrm{GHz}$, the currents on the top and bottom Four-U-SRRs are in the same direction. On the contrary, at 6.3 GHz, the currents on the top and bottom are in the opposite direction. According to the current distribution, we can analyze the induced magnetic field. Figure 4 shows that the $\mathrm{x}$ component of the induced magnetic field $\mathrm{H}_{\mathrm{x}}$ is nonzero. And moreover, at $5.1 \mathrm{GHz}, \mathrm{H}_{\mathrm{x}}$ and $\mathrm{E}_{\mathrm{x}}$ are in the opposite directions, while at $6.3 \mathrm{GHz}$, they are in the same direction. This causality between electric and magnetic fields is consistent with the constitute equation Eq. (1).

In summary, by twisting the four "U" SRRs in the propagation direction, which owns the fourfold rotational symmetry, a uniaxial CMM can be constructed. We study the structure via the numerical simulation and the experiment at 3.5 $-7.5 \mathrm{GHz}$. The simulation results agree with the experimental results very well. This artificial structure gives us so strong chirality that the refractive index of RCP or LCP can be pushed to be negative at the vicinity of the resonance.
Such structure can be scaled to other frequencies. The proposed chiral structures are suitable for planar fabrication. In the future, we can use this planar design to contracture bulk CMMs via layer-by-layer method.

This work is supported by the European Union under the projects EU-PHOME and EU-ECONAM, and TUBITAK under the Project Nos. 107A004 and 107A012. One of the authors (E.O.) also acknowledges partial support from the Turkish Academy of Sciences. Rongkuo Zhao acknowledges the China Scholarship Council (CSC) for financial support.

${ }^{1}$ J. B. Pendry, Science 306, 1353 (2004).

${ }^{2}$ S. Tretyakov, I. Nefedov, A. Sihvola, S. Maslovski, and C. Simovski, J. Electromagn. Waves Appl. 17, 695 (2003).

${ }^{3}$ A. V. Rogacheva, V. A. Fedotov, A. S. Schwanecke, and N. I. Zheludev, Phys. Rev. Lett. 97, 177401 (2006).

${ }^{4}$ B. Wang, J. Zhou, T. Koschny, and C. M. Soukoulis, Appl. Phys. Lett. 94, 151112 (2009).

${ }^{5}$ S. Zhang, Y.-S. Park, J. Li, X. Lu, W. Zhang, and X. Zhang, Phys. Rev. Lett. 102, 023901 (2009).

${ }^{6}$ E. Plum, J. Zhou, J. Dong, V. A. Fedotov, T. Koschny, C. M. Soukoulis, and N. I. Zheludev, Phys. Rev. B 79, 035407 (2009).

${ }^{7}$ J. Zhou, J. Dong, B. Wang, T. Koschny, M. Kafesaki, and C. M. Soukoulis, Phys. Rev. B 79, 121104(R) (2009).

${ }^{8}$ M. Decker, M. Ruther, C. E. Kriegler, J. Zhou, C. M. Soukoulis, S. Linden, and M. Wegener, Opt. Lett. 34, 2501 (2009).

${ }^{9}$ J. D. Jackson, Classical Electrodynamics, 3rd ed. (Wiley, New York, 1998).

${ }^{10}$ J. A. Kong, Electromagnetic Wave Theory (EMW, Cambridge, 2008).

${ }^{11}$ C. Enkrich, M. Wegener, S. Linden, S. Burger, L. Zschiedrich, F. Schmidt, J. F. Zhou, T. Koschny, and C. M. Soukoulis, Phys. Rev. Lett. 95, 203901 (2005).

${ }^{12}$ S. Linden, C. Enkrich, M. Wegener, J. Zhou, T. Koschny, and C. M. Soukoulis, Science 306, 1351 (2004).

${ }^{13}$ I. Sersic, M. Frimmer, E. Verhagen, and A. F. Koenderink, Phys. Rev. Lett. 103, 213902 (2009).

${ }^{14}$ H. Liu, D. A. Genov, D. M. Wu, Y. M. Liu, Z. W. Liu, C. Sun, S. N. Zhu, and X. Zhang, Phys. Rev. B 76, 073101 (2007).

${ }^{15}$ N. Liu, H. Liu, S. Zhu, and H. Giessen, Nat. Photonics 3, 157 (2009).

${ }^{16}$ D. H. Kwon, D. H. Werner, A. V. Kildishev, and V. M. Shalaev, Opt. Express 16, 11822 (2008).

${ }^{17}$ C. Menzel, C. Rockstuhl, T. Paul, and F. Lederer, Appl. Phys. Lett. 93, 233106 (2008).

${ }^{18}$ N. Liu and H. Giessen, Opt. Express 16, 21233 (2008).

${ }^{19}$ X. Xiong, W. H. Sun, Y. J. Bao, M. Wang, R. W. Peng, C. Sun, X. Lu, J. Shao, Z. F. Li, and N. B. Ming, Phys. Rev. B 81, 075119 (2010).

${ }^{20}$ R. Zhao, T. Koschny, E. N. Economou, and C. M. Soukoulis, Phys. Rev. B 81, 235126 (2010). 